\title{
Autosomal dominant progressive nephropathy with hypertension
}

INSERM

\section{Source}

INSERM. (1999). Orphanet: an online rare disease and orphan drug data base. Autosomal dominant progressive nephropathy with hypertension. ORPHA:88659

A rare, genetic hypertension characterized by an adult onset of increased blood pressure associated with nephropathy progressing to end-stage renal disease. Renal biopsy may show interstitial fibrosis, glomerulosclerosis and mild tubular atrophy. Increased serum creatinine and proteinuria have also been reported. 